\title{
Más que humanos: el robot en la ciencia ficción
}

\author{
Mario César Arizmendi Guzmán
}

\section{Resumen}

La inteligencia artificial tiene un papel importante dentro de la ciencia ficción. El presentar androides, autómatas y robots como simples máquinas que cumplen una tarea específica deja de ser relevante cuando empiezan a actuar de forma errática, comportándose como seres humanos. ¿Por qué pueden crear, sentir y actuar como una persona común y corriente? Con base en esta cuestión se analiza, desde una perspectiva literaria, el arquetipo del robot más que humano dentro de la ciencia ficción.

Palabras clave: inteligencia artificial, robots, ciencia ficción, humanidad, representación.

\section{MORE THAN HUMANS: THE ROBOT IN SCIENCE FICTION}

\begin{abstract}
Artificial Intelligence has an important role in science fiction. The presentation of androids, automatons and robots as simple machines that fulfill a task stops being relevant when they begin to act erratically, when they begin to act as human beings. Why are they able to create, feel and act as an ordinary person? Based on this issue we will analize, from a literary perspective, the robot as something more than human archetype.
\end{abstract}

Keywords: artificial Intelligence, robots, science fiction, humanity, representation.

Dol: http://doi.org/10.22201/codeic.16076079e.2020.v21n2.a10. 


\section{Mario César Arizmendi Guzmán}

arizmendi.mario@gmail.com https://orcid.org/0000-0003-3193-8678

Estudió Lengua y Literaturas Modernas en la Facultad de Filosofía y Letras de la Universidad Nacional Autónoma de México (UNAM). Actualmente colabora con la Revista Digital Universitaria. Se dedica a la escritura y a la literatura. Sus áreas de interés son la lingüística, la semiótica y la traducción. 
My name is Ozymandias, king of kings:

Look on my works, ye Mighty, and dispair!

David, Alien Covenant ${ }^{1}$

\section{Introducción}

Hoy en día es difícil encontrar a una persona que no sepa qué es un robot. El mundo de las artes se ha empeñado, desde hace años, en presentarlos como máquinas súper desarrolladas con características envidiables: resistencia, fuerza, adaptabilidad, trascendencia, inmortalidad. No obstante, estos rasgos no son, necesariamente, lo más Ilamativo. Una aproximación y análisis de este tema puede adentrar al lector a una nueva forma de profundizar las obras de ciencia ficción.

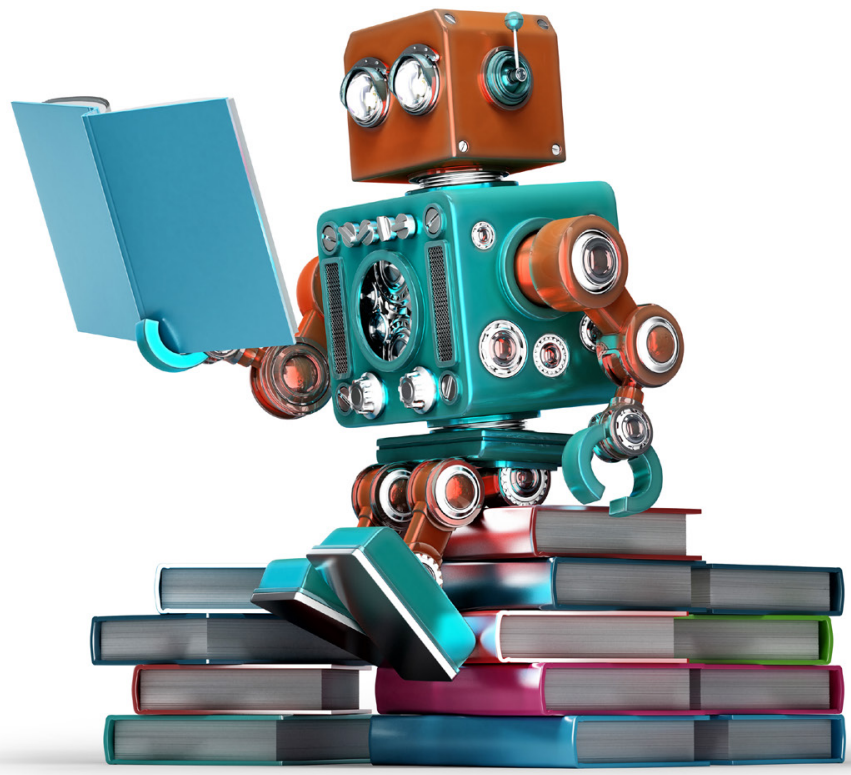

' El fragmento corresponde al poema, Ozymandias (1818), de Percy Byshee Shelley.
La inteligencia artificial podría considerarse ajena a la vida y la naturaleza por su condición de máquina. A pesar de ello, dentro de la ciencia ficción no se muestran como los otros, ya que, sin ser humanos, tienen características, en teoría, exclusivas de nuestra especie, ¿cómo es esto posible? Para contestar esta pregunta, en este texto analizaré brevemente, desde mi formación en literatura y mi gusto por este género literario, el arquetipo del robot más que humano dentro de la ciencia ficción. 


\section{El ser humano no es sinónimo de humanidad}

¿Qué significa ser humano? De acuerdo con la Real Academia Española, dicha definición se resume y se limita al hombre como ser racional. Sin embargo, la cuestión es mucho más compleja. ${ }^{2}$ Una manera de llegar a una definición propia sería destacar una serie de cualidades (o defectos) que nos hacen humanos. Por ejemplo, se considera humana la habilidad de sentir, de aprender y de crear. Una persona tiene funciones cognitivas y es consciente de sí misma, capaz de diferenciar y elegir entre cosas ajenas a ella.

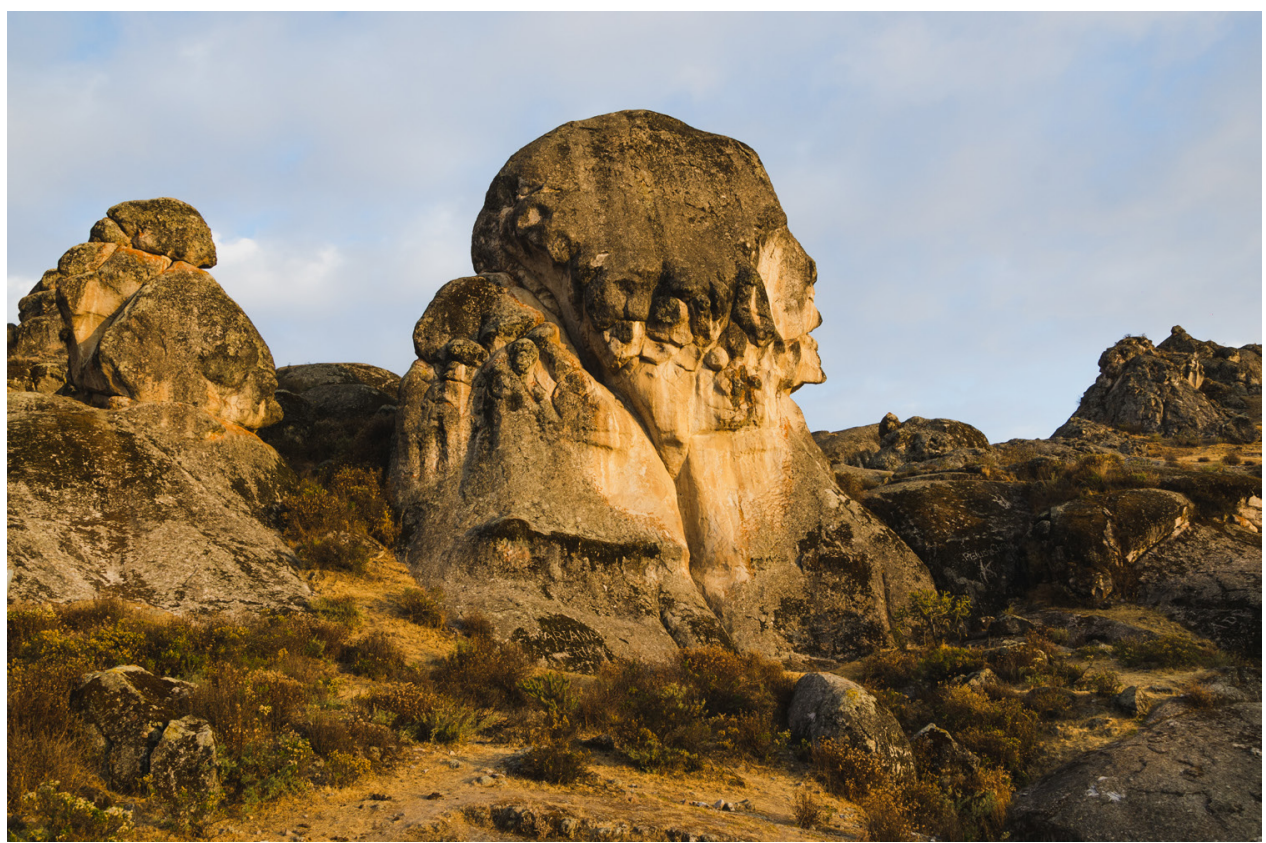

Por otra parte, habría que pensar qué elementos podrían considerarse como no humanos. El hombre se encuentra limitado por su estado físico, lo que significa que puede cansarse, enfermarse y morir. Aunque ya se señaló su capacidad de aprender, hacerlo requiere de mucho tiempo en la mayoría de los casos $y$, a veces, sólo se obtienen resultados modestos. A pesar de lo contrastantes que pudieran parecer estos aspectos, físicos y cognitivos, ambos mantienen

2 Desde Sócrates, Platón y Aristóteles, con Kant, Hegel y Heidegger en medio, y hasta las últimas corrientes filosóficas como el personalismo, se ha intentado definir al hombre con resultados igual de variados en los miles de años de intentar contestar esta pregunta. equilibrio dentro del ser humano. Pero ¿qué pasaría si se creara una máquina que potenciara nuestras virtudes y careciera de nuestros defectos?

Además, faltaría definir humanidad, no como especie, sino como condición: tener humanidad. Para fines prácticos, resulta todo lo que nos diferencia de los animales y las máquinas. La humanidad recae en conceptos como la sensibilidad, la empatía, la bondad, entre otros, aunque también se deberían incorporar elementos considerados perjudiciales, como la crueldad y la negatividad. 
Basta con escuchar las noticias, salir a la calle, prestarle atención a la forma en que nos relacionamos con los demás, para notar que los individuos están perdiendo su empatía. No es que se vuelvan malvados, sino que se alienan aún más de su entorno y se vuelven indiferentes ante él. Al respecto podemos retomar que "Cuando se habla del futuro (incierto e imposible) en literatura prospectiva, usado como motivo, sólo se habla del miedo al presente por razones de alienación social e individual" (J. I. Ferreras, cit. por Moreno, 2008, p. 148). Una de las razones que podría explicar este comportamiento recae en el uso excesivo de la tecnología. De una manera cínica y creativa resulta irónico que, a partir de una de las cumbres de la tecnología, en este caso la inteligencia artificial, en el arte se manifiesten, constantemente, las tantas similitudes entre la máquina y el hombre.

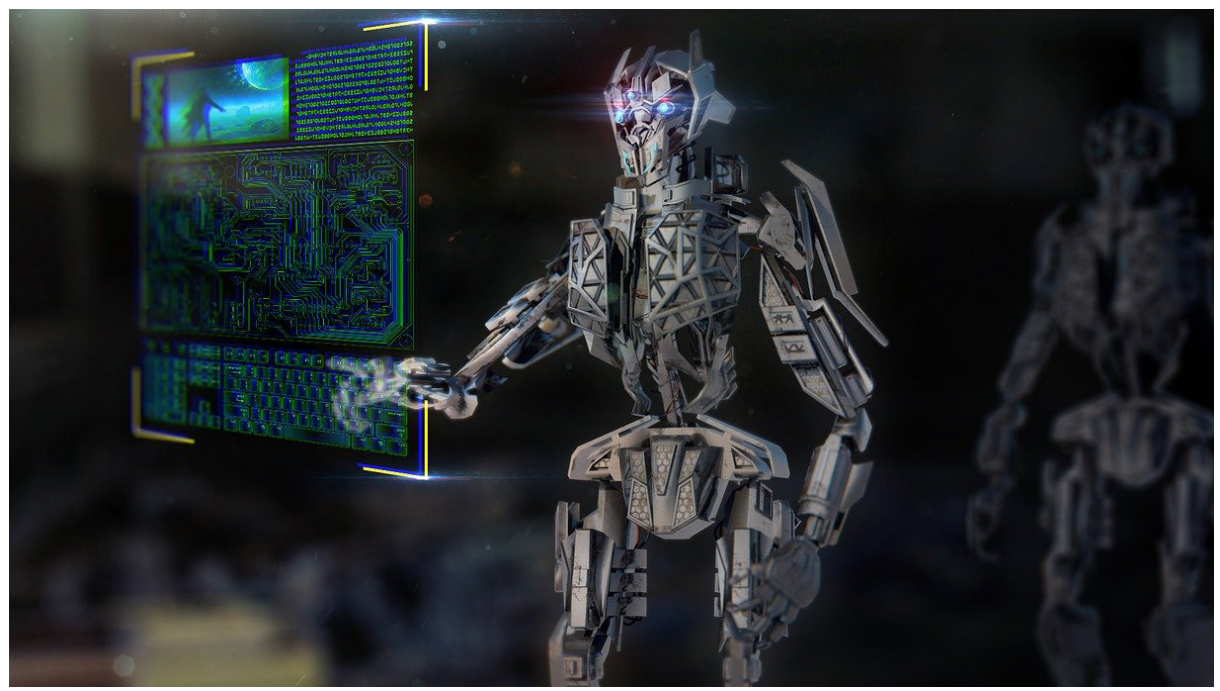

\section{Inteligencia artificial y robots}

En pocas palabras, la inteligencia artificial es el mecanismo desarrollado para que las máquinas "piensen". Si bien esta definición parece escueta, podemos pensar que el robot representa la forma física (hardware) y la inteligencia artificial, el programa (software), y en conjunto crean una máquina con un propósito definido. Cabe destacar que éstas son, o pretenden ser, la antítesis de la humanidad, pues no presentan características humanas a menos que estén contempladas en su programación y aun así encuentran limitaciones en lo que un hombre pudiera llegar a ser y hacer. Sin embargo, en múltiples ejemplos, el arte propone que, en un futuro tal vez no muy lejano, la máquina tomará el lugar del hombre.

Y no sólo se trata de una propuesta, el ideal de suplantar al hombre con una máquina de figura humanoide procede desde mucho tiempo atrás, tal y como Kagarlitski (1974) lo describe: "Cuando los hombres soñaban con una técnica universal para el futuro, se la imaginaban antropomorfa, lo mismo que los antiguos dioses estaban hechos a su imagen y semejanza. Creían que al hombre 
le debería sustituir un hombre artificial, mecánico" (p. 125). Este concepto no sólo remarca el ego de la humanidad al querer igualarse a los dioses y que sus creaciones estén a la par de ellos; también habla del anhelo milenario de componer una máquina que suceda a la humanidad. Además, nos señala la forma en que hace las cosas: siempre como una imitación de sí mismo, lo que da como resultado, al robot antropomorfo.

Pese a que los robots antropomorfos son un vehículo mediante el cual se mueve la inteligencia artificial, ésta no se encuentra atada a su condición física. Kagarlitski nos menciona que:

Pronto se vio que los robots no tenían por qué conservar necesariamente su forma antropomorfa. Esta forma expresa el carácter multifuncional [...], la capacidad de elección, voluntad propia, en una palabra, caracteriza la personalidad. La forma antropomorfa no es sino simbólica. Por eso cuando pueden prescinden de ella (1974, p. 130).

De este modo, se puede hablar de inteligencia artificial sin que obligatoriamente se trate de robots. Esta modalidad se extiende al mundo real, como sucede con las asistentes digitales Alexa, Cortana o Siri, y al plano de la ciencia ficción, donde uno de los ejemplos más notables sería Hall 9000, de 2001: Una odisea espacial, la supercomputadora que no posee una forma antropomorfa.

La inteligencia artificial es un hecho con el que convivimos a diario, aunque no del modo exacto cómo se ha planteado en muchos libros o películas. Sin embargo, estas máquinas no nos interesan en este artículo, sino, esa visión irreal que gira entorno a ellas, la que prolifera en la ciencia ficción.

\section{La ciencia ficción: el futuro presente}

Siempre hay problemas para definir un género literario y la ciencia ficción no se escapa de ello. Debido a la variedad de temas que aborda, no se caracteriza por algo en específico, pero la inteligencia artificial ha tomado protagonismo en este ámbito, a través de la literatura, el cine, la escultura, así como otras manifestaciones artísticas. A veces, se codea con la vida extraterrestre, como en la obra de Ted Chiang de 1996, La historia de tu vida; otras, con los viajes en el tiempo, en El fin de la eternidad (1955) de Isaac Asimov; o con la vida en el espacio, en la saga Los cantos de Hyperion (1989-1997) de Dan Simmons, etcétera. Resulta necesario destacar cómo en muchas ocasiones todos estos temas se juntan para crear una obra tan diversa y compleja, como la space opera por antonomasia, Star Wars (1977-2019).

Entonces, ¿de qué trata la ciencia ficción? Dada su variedad, podríamos afirmar que es sobre temas futuristas. En tal caso, tal vez su carácter imposible provoque que no se le tome completamente en serio. No obstante, es muy sencillo que se pierda de vista que las obras dentro de este género tratan del hombre. Al respecto, dice Fernando Ángel Moreno (2004), que "La mayor 
Esta frase no tiene relación alguna con la novela Más que humano (1953), del estadounidense Theodor Sturgeon. El argumento de ésta recae en un grupo de personas con poderes sobrenaturales y que, por lo mismo, no encajan completamente en la sociedad.

A pesar de que sí se trata de una novela de ciencia ficción, no se encuentra dentro del apartado

que para este artículo atañe.

${ }^{4}$ Las leyes de la robótica, concebidas por Isaac Asimov son: 1) un robot no puede dañar a un humano ni permitir que sufran daño, 2) debe obedecer a los humanos, excepto cuando esto esté en contra de la primera ley, y 3) el robot debe protegerse, a menos que esto vaya en contra de la primera o la segunda ley. parte de la buena ciencia ficción trata sobre filosofía, o al menos, profundiza en cuestiones fundamentales de la cultura humana" (p. 66).

Existe una condición que afecta a todas las obras de ficción y que evita que sean muy diferentes entre sí: todas son creaciones del hombre. Esto quiere decir que está limitado por su propia realidad y sólo puede replicarla. Sobre el tema, Moreno confirma (2008) que "Demasiado a menudo cuando hablamos de novelas de robots, decimos: en realidad, hablan de robots. No se recuerda que cualquier ser humano sólo puede escribir sobre seres humanos" (p. 73). Por eso no interesa cuántos seres fantásticos presenten El señor de los anillos o Juego de tronos, en realidad, la sociedad de la que hablan está basada en cualquier modelo típico de la Edad Media. En las novelas prospectivas que nos atañen, tampoco es fundamental lo inimaginable de los avances tecnológicos ni de los rasgos humanos: los temas que se abordan siguen siendo de una actualidad que muchas veces da miedo.

\section{Más que humanos 3}

Dentro de la ciencia ficción, la inteligencia artificial suele tener el rol de antagonista. Ya sea por su programación, como es el caso del T-800 en Terminator, o bien, por una falla en ésta, como la que tiene Dolores en Westworld. También se puede destacar un seguimiento erróneo de Las tres leyes de la robótica, ${ }^{4}$ como en algunos de los relatos de Yo, robot.

No obstante, hay ejemplos en donde el robot es bueno. Por mencionar un par de casos, en Wall-E o en El hombre bicentenario, por alguna causa externa, como la soledad en Wall-E o el amor de un ser humano en Andrew, los "robots" desarrollan una personalidad que se destaca por mantener valores benévolos y protectores.

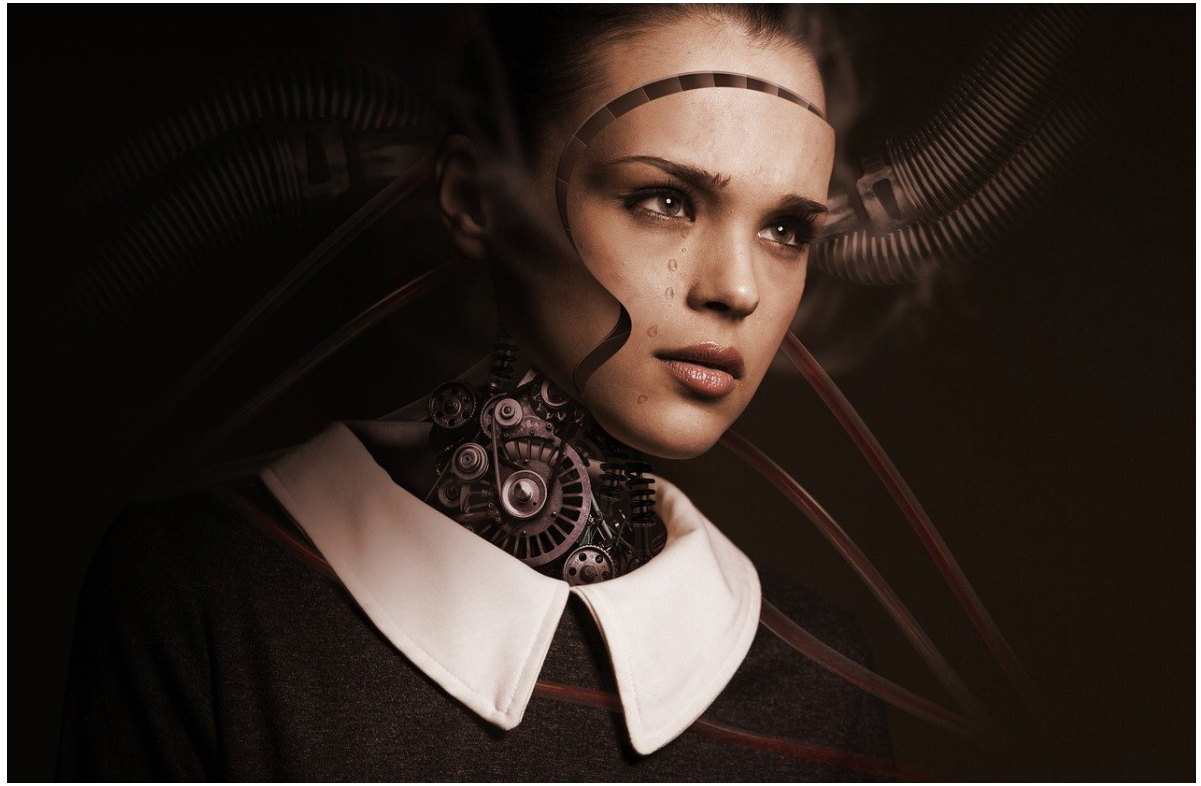


${ }^{5}$ Estoy consciente de la existencia de robots dentro de la cultura popular, que no necesariamente encajan en este patrón. Existe el caso del Omnidroide X10 de Los increíbles (2004); sin embargo, tanto el tema de la película, los superhéroes, y lo que busca destacar, la familia, son diferentes a lo que la ciencia ficción busca con la inteligencia artificial.
Sin embargo, tanto los robots "buenos" como los "malos" comparten una cualidad que también se desarrolla: adquirir rasgos que podemos considerar esencialmente humanos. ${ }^{5}$ La inteligencia artificial sufre un fallo en su programación y comienza a manifestar características humanas, como la capacidad de crear, de sentir, de diferenciar, de elegir y hasta de emitir juicios.

Entre todas estas características, se distingue una: amar la vida por sobre todas las cosas. Tal vez éste sea el rasgo más importante que adquieren los robots, muchas veces sin importar que sean los héroes o villanos de la historia. como ocurre en los siguientes casos:

En la película de Blade Runner, los replicantes luchan por sobrevivir y hacen cualquier cosa para conseguirlo. Al final, cuando Deckard está a punto de morir, Roy Batty, el androide principal, lo salva y ejecuta uno de los pasajes más bellos de la ciencia ficción. Pero ¿por qué sucede esto? Precisamente debido a que el replicante ya no tiene nada que perder; en comparación, Deckard podía dejar de existir, situación que Batty entiende, lo cual parece probar que el androide es más humano que el humano mismo.

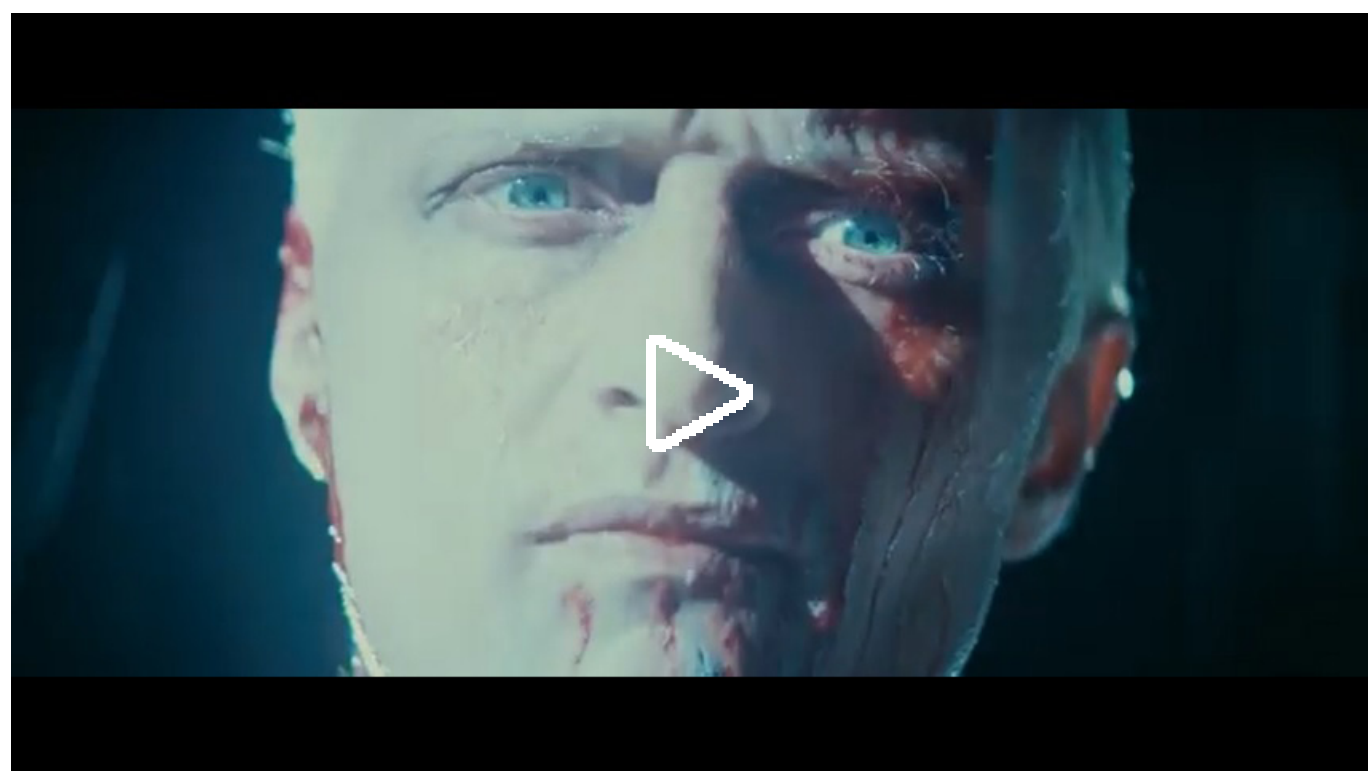

Para retomar el ejemplo de Hall 9000, la supercomputadora omnipresente en la nave Discovery no tiene una forma humana, pero sí presenta rasgos de humanidad. En el momento en que ésta se pone a cantar, lo hace con el propósito de calmarse antes su muerte (o desconexión, según se vea), pero, especialmente, para suplicar por continuar existiendo. 


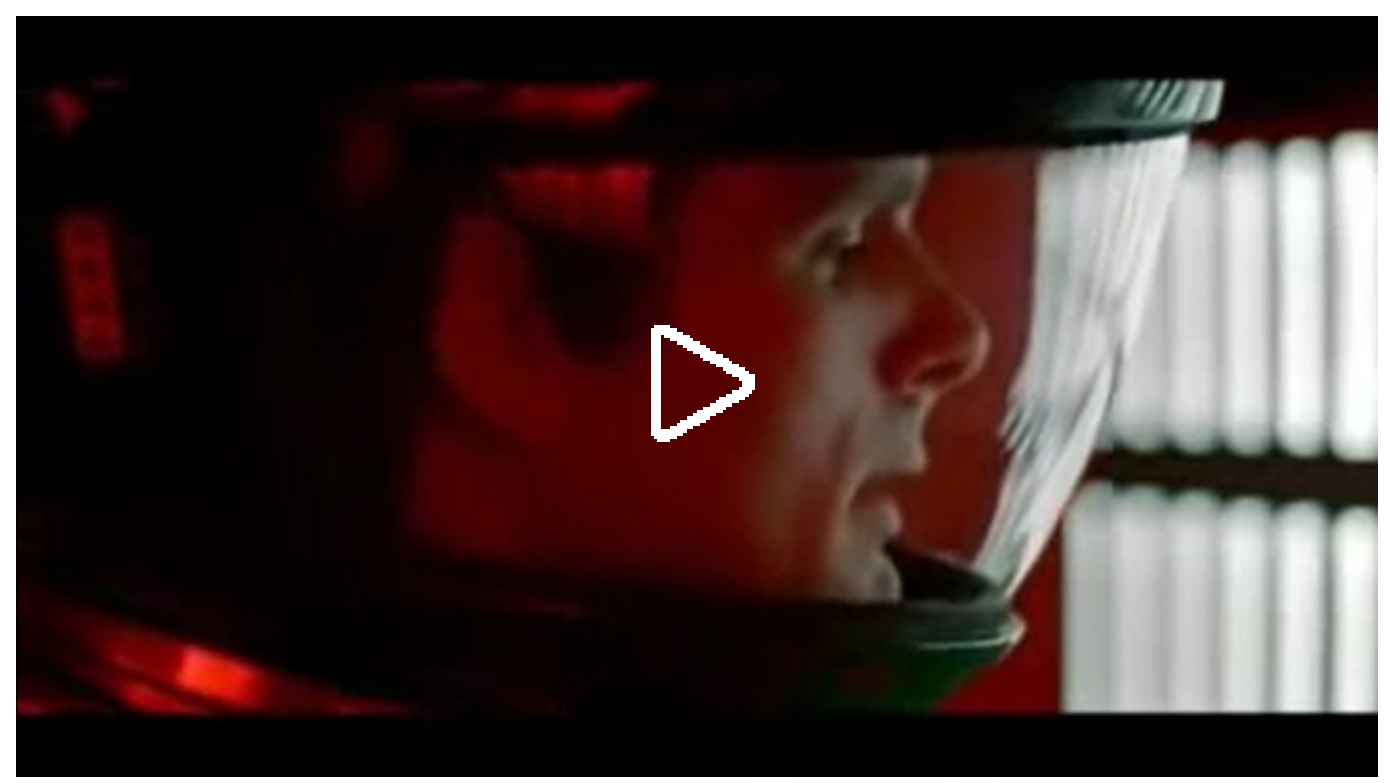

Otro ejemplo de amor a la vida se puede apreciar en la película Chappie (2015), del director Neill Blomkamp. En ella, no se trata del respeto hacia la vida o una especie de amor propio como en los casos anteriores, sino del aprecio a una persona. El robot se enfurece cuando matan a la mujer que lo crió, Yolandi, por lo cual emprende una cacería contra su asesino.

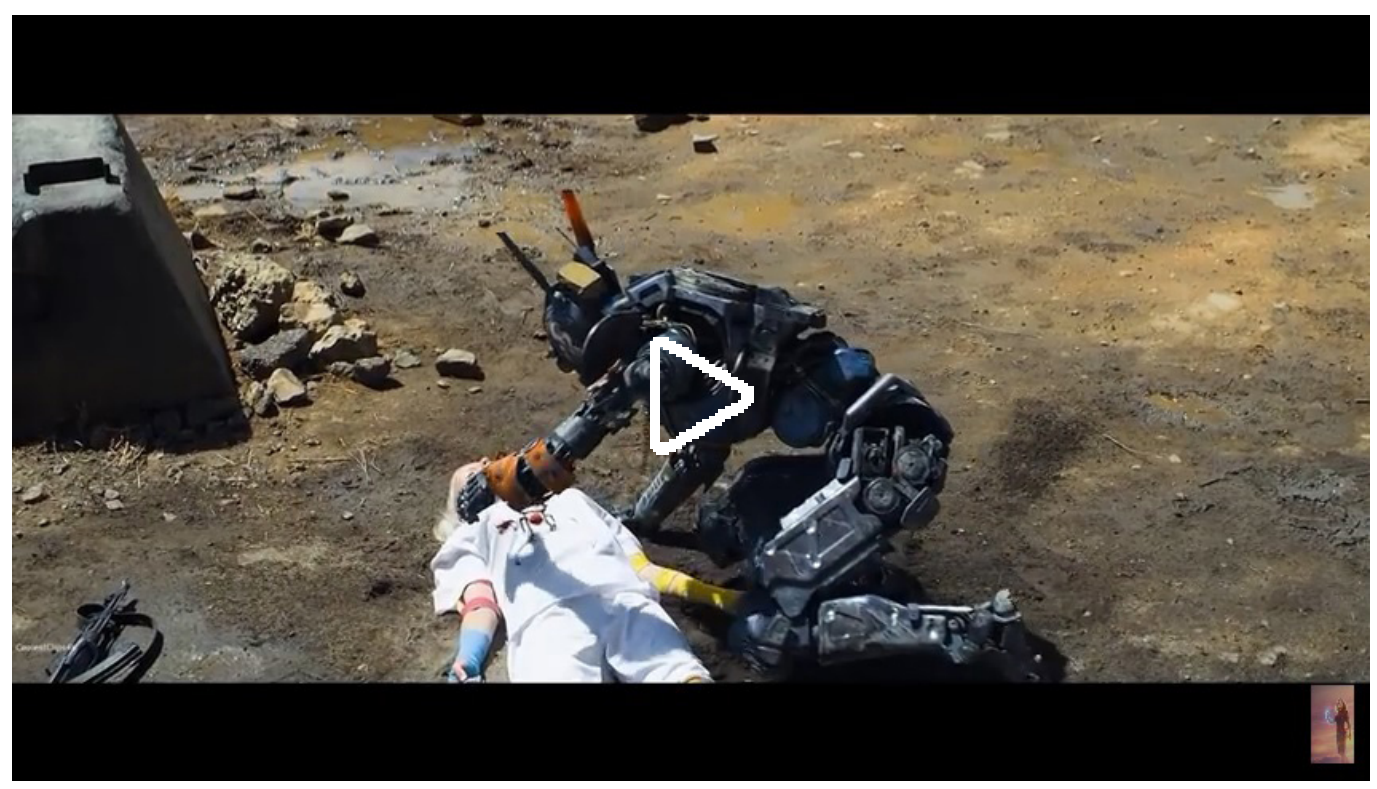

Los tres ejemplos resaltan el aprecio hacia la vida, la resignación, el miedo, la súplica, la tristeza, la ira; cuestiones afines a los humanos, no a las máquinas. 


\section{Conclusiones}

Una de las fórmulas que más éxito ha cosechado dentro del mundo del arte es la del robot demasiado humano. Cada año, este tema forma parte de la cartelera de cine o constituye la obra literaria ganadora del premio Hugo o Nébula, ¿por qué? Simplemente ya que el hombre necesita que se le recuerde constantemente su humanidad. Si no, ¿existiría otra razón? Quizá la tecnología nos aliena cada vez más, absorbe nuestros espacios y tiempo, y nos hace olvidar la convivencia frente a frente; sin embargo, el robot no es el otro, nosotros lo somos al actuar de forma mecánica, volviéndonos insensibles ante el entorno. Curiosamente, para apreciar la vida, el arte nos muestra que aquellas máquinas, robots o inteligencias artificiales que no pueden hacerlo per se son quienes más lo hacen.

Así, quedan abiertas las preguntas: ¿qué tanta humanidad desarrollan las máquinas?, ¿acaso podría tratarse tan sólo de un fallo en su programación? Creo que la segunda premisa no excluye a la primera, pues, ¿qué cuestión más afín al hombre que equivocarse constantemente? En tal caso, un desenvolvimiento humano se puede presentar, en historias de ciencia ficción, de forma legítima.

Por mi parte, elijo pensar que la actualidad de esas obras reside en el reconocimiento de nuestra humanidad, de nuestro ser humano, a partir de la visión de los otros. El que la inteligencia artificial y los robots sean capaces de amar la vida por la vida misma es lo que los convierte en más que humanos.

\section{Referencias}

* Alonso Burgos, Jesús (2017). Teoría e historia del hombre artificial. De autómatas, ciborgs, clones y otras criaturas. Akal.

Asimov, Isaac (2009). El hombre bicentenario. Cuentos completos II. Zeta Bolsillo.

- Asimov, Isaac (1991). Robot visions. Roc.

- Auerbach, Erich (2014). Mimesis. La representación de la realidad en la literatura occidental. Fondo de cultura económica.

* Blomkamp, Neil (director). (2015). Chappie [película]. Media Rights Capital.

* Clarke, Arthur C. (2018). Una odisea espacial. La saga completa. Debolsillo.

Dick, Philip K. (2017). ¿Sueñan los androides con ovejas eléctricas? Cátedra.

* Kagarlitski, Yuli (1974). ¿Qué es la ciencia ficción? Guadarrama.

* Kayser, Wolfgang (1992). Interpretación y análisis de la obra literaria. Gredos. 
* Kubrick, Stanley (director). (1968). 2001: Odisea del espacio [película]. MetroGoldwyn-Meyer.

* Moreno, Pedro Ángel (2008). La ficción proyectiva: propuesta para una delimitación del género de la ciencia ficción. En Pellisa Teresa López y Fernando Ángel Moreno Serranos (Eds.), Ensayos sobre ciencia ficción y literatura fantástica (pp.). Universidad Carlos III.

* Moreno, Pedro Ángel (2010). Teoría de la literatura de ciencia ficción. Poética y retórica de lo prospectivo. Portal Editions.

* Muñoz Corcuera, Alfonso (2008) La ética robótica de Asimov. En Pellisa Teresa López y Fernando Ángel Moreno Serranos (Eds.), Ensayos sobre ciencia ficción y literatura fantástica (pp.). Universidad Carlos III,

* Reynolds, Alastair (2006). Zima Blue and other stories. Night Shade Books.

* Rómar, Antonio (2008) El mito y la ciencia ficción. La imaginación en el proceso de construcción de lo real. En Pellisa Teresa López y Fernando Ángel Moreno Serranos (Eds,), Ensayos sobre ciencia ficción y literatura fantástica (pp.). Universidad Carlos III.

- Scott, Ridley (director). (1982). Blade Runner [película]. The Ladd Company.

\section{Sitios de interés}

* Ciencia ficción

Inteligencia artificial

- Leyes de la robótica

Blade Runner

2001

- Chappie

\section{Cómo citar este artículo}

* Arizmendi Guzmán, Mario César (2020). Más que humanos: el robot en la ciencia ficción. Revista Digital Universitaria (RDU). Vol. 21, núm. 2 marzo-abril. Dol: http://doi. org/10.22201/codeic.16076079e.2020.v21n2.a10.

Recepción: 11/12/2019. Aprobación: 11/02/2020 